Article

\title{
The Spectral Response of the Landsat-8 Operational Land Imager
}

\author{
Julia A. Barsi ${ }^{1, *}$, Kenton Lee ${ }^{2}$, Geir Kvaran ${ }^{2}$, Brian L. Markham ${ }^{3}$ and Jeffrey A. Pedelty ${ }^{3}$ \\ 1 Science Systems and Applications, Inc., NASA/GSFC Code 618, Greenbelt, MD 20771, USA \\ 2 Ball Aerospace \& Technology Corp., 1600 Commerce Street, Boulder, CO 80301, USA; \\ E-Mails: klee@ball.com (K.L.); gkvaran@ball.com (G.K.) \\ 3 NASA/GSFC Code 618, Greenbelt, MD 20771, USA; E-Mails: brian.1.markham@nasa.gov (B.L.M.); \\ jeffrey.a.pedelty@nasa.gov (J.A.P.) \\ * Author to whom correspondence should be addressed; E-Mail: julia.barsi@nasa.gov; \\ Tel.: +1-301-614-6667; Fax: +1-301-614-6695.
}

External Editors: James C. Storey, Ron Morfitt and Prasad S. Thenkabail

Received: 29 July 2014; in revised form: 4 October 2014 / Accepted: 9 October 2014 /

Published: 23 October 2014

\begin{abstract}
This paper discusses the pre-launch spectral characterization of the Operational Land Imager (OLI) at the component, assembly and instrument levels and relates results of those measurements to artifacts observed in the on-orbit imagery. It concludes that the types of artifacts observed and their magnitudes are consistent with the results of the pre-launch characterizations. The OLI in-band response was characterized both at the integrated instrument level for a sampling of detectors and by an analytical stack-up of component measurements. The out-of-band response was characterized using a combination of Focal Plane Module (FPM) level measurements and optical component level measurements due to better sensitivity. One of the challenges of a pushbroom design is to match the spectral responses for all detectors so that images can be flat-fielded regardless of the spectral nature of the targets in the imagery. Spectral variability can induce striping (detector-to-detector variation), banding (FPM-to-FPM variation) and other artifacts in the final data products. Analyses of the measured spectral response showed that the maximum discontinuity between FPMs due to spectral filter differences is $0.35 \%$ for selected targets for all bands except for Cirrus, where there is almost no signal. The average discontinuity between FPMs is $0.12 \%$ for the same targets. These results were expected and are in accordance with the OLI requirements. Pre-launch testing identified
\end{abstract}


low levels (within requirements) of spectral crosstalk amongst the three $\mathrm{HgCdTe}$ (Cirrus, SWIR1 and SWIR2) bands of the OLI and on-orbit data confirms this crosstalk in the imagery. Further post-launch analyses and simulations revealed that the strongest crosstalk effect is from the SWIR1 band to the Cirrus band; about $0.2 \%$ of SWIR1 signal leaks into the Cirrus. Though the total crosstalk signal is only a few counts, it is evident in some scenes when the in-band cirrus signal is very weak. In moist cirrus-free atmospheres and over typical land surfaces, at least $30 \%$ of the cirrus signal was due to the SWIR1 band. In the SWIR 1 and SWIR2 bands, crosstalk accounts for no more than $0.15 \%$ of the total signal.

Keywords: Landsat-8; OLI; spectral response; RSR; characterization

\section{Introduction}

The Operational Land Imager (OLI) is the latest instrument in the Landsat series of satellite imagers, launched aboard the Landsat- 8 in February 2013. The OLI continues the legacy of Landsat, building the archive of moderate resolution earth imagery, but the instrument itself is significantly different than the Thematic Mapper (TM) series of sensors aboard Landsat-5 and -7. The TM instruments were whiskbroom sensors with relatively few detectors sweeping over the earth in the cross-track direction of the satellite. The OLI is a pushbroom sensor, with long arrays of detectors forming the image as the satellite moves across the Earth [1]. The OLI also includes two bands that are not on the TMs; a Cirrus band to aid in detection of cirrus clouds and a Coastal/Aerosol (CA) band for better resolution of water and aerosols in the blue region. Unlike the TMs, OLI does not include a thermal band. The Thermal Infrared Sensor (TIRS) covers the thermal region and has two bands [2]. TIRS characteristics are not covered in this paper. Table 1 shows the spectral band characteristics of the OLI alongside the reflective bands of the Landsat-7 Enhanced Thematic Mapper Plus (ETM+).

Table 1. Landsat-8 Operational Land Imager (OLI) spectral band requirements as compared to Landsat-7 ETM+.

\begin{tabular}{ccccc}
\hline Band Name & $\begin{array}{c}\text { Landsat-8 OLI } \\
\text { Maximum Bandpass } \\
(\boldsymbol{\mu m})\end{array}$ & $\begin{array}{c}\text { Ground } \\
\text { Sample } \\
\text { Distance }(\mathbf{m})\end{array}$ & $\begin{array}{c}\text { Landsat-7 ETM+ } \\
\text { Bandpass }(\boldsymbol{\mu m})\end{array}$ & $\begin{array}{c}\text { Ground Sample } \\
\text { Distance (m) }\end{array}$ \\
\hline Coastal/Aerosol (CA) & $0.433-0.453$ & 30 & & \\
Blue & $0.450-0.515$ & 30 & $0.450-0.515$ & 30 \\
Green & $0.525-0.600$ & 30 & $0.525-0.605$ & 30 \\
Red & $0.630-0.680$ & 30 & $0.630-0.690$ & 30 \\
NIR & $0.845-0.885$ & 30 & $0.775-0.900$ & 30 \\
SWIR1 & $1.560-1.660$ & 30 & $1.550-1.750$ & 30 \\
SWIR2 & $2.100-2.300$ & 30 & $2.090-2.350$ & 30 \\
PAN & $0.500-0.680$ & 15 & $0.520-0.900$ & 15 \\
Cirrus & $1.360-1.390$ & 30 & & \\
\hline
\end{tabular}


The spectral response characteristics of the OLI instrument, like any remote sensing instrument, are key to understanding and utilizing the data. This paper describes: (1) how the spectral characterization was performed prior to launch; (2) presents a summary of the spectral response data; (3) provides links to the complete data sets available online; (4) shows some implications of the variation in spectral response across the instrument's field of view on the uniformity of the image data; and (5) discusses a weak artifact that is visible in the OLI Cirrus data and shows how it is related to the design and spectral response of the OLI. Portions of the content of this paper have been previously presented at conferences and published in their proceedings [3].

The OLI pushbroom design uses long arrays of detectors to cover the 15 degree field of view (185 km swath) [1]. A four-mirror telescope focuses incoming light on the focal plane. The detectors are divided between 14 Focal Plane Modules (FPMs) (Figure 1). Each module includes a linear array of detectors for each band, which is covered by the spectral filters to differentiate the spectral bands. A multi-spectral (MS) FPM is 494 detectors wide (988 detectors in the Pan band) so each MS band consists of 6916 distinct imaging detectors. The visible and near-infrared (VNIR) spectral bands use Silicon (Si) PIN photodiodes and the shortwave infrared (SWIR) bands use Mercury-Cadmium-Telluride (HgCdTe) photodiodes.

Figure 1. A photograph of the completed OLI focal plane assembly, with the FPM numbers added. The optical axis runs through the center of the modules, between the odd number FPMs and the even number FPMs. The colored bars are the filter sticks for each band on each module. The detectors are behind the filter sticks. The band order of the filters from most off-axis to least off-axis is: Cirrus, SWIR1, SWIR2, Green, Red, NIR, CA, Blue, Pan, such that on the assembled focal plane the Pan band arrays are closest together in the odd/even FPM pairs, and the Cirrus bands are furthest away.

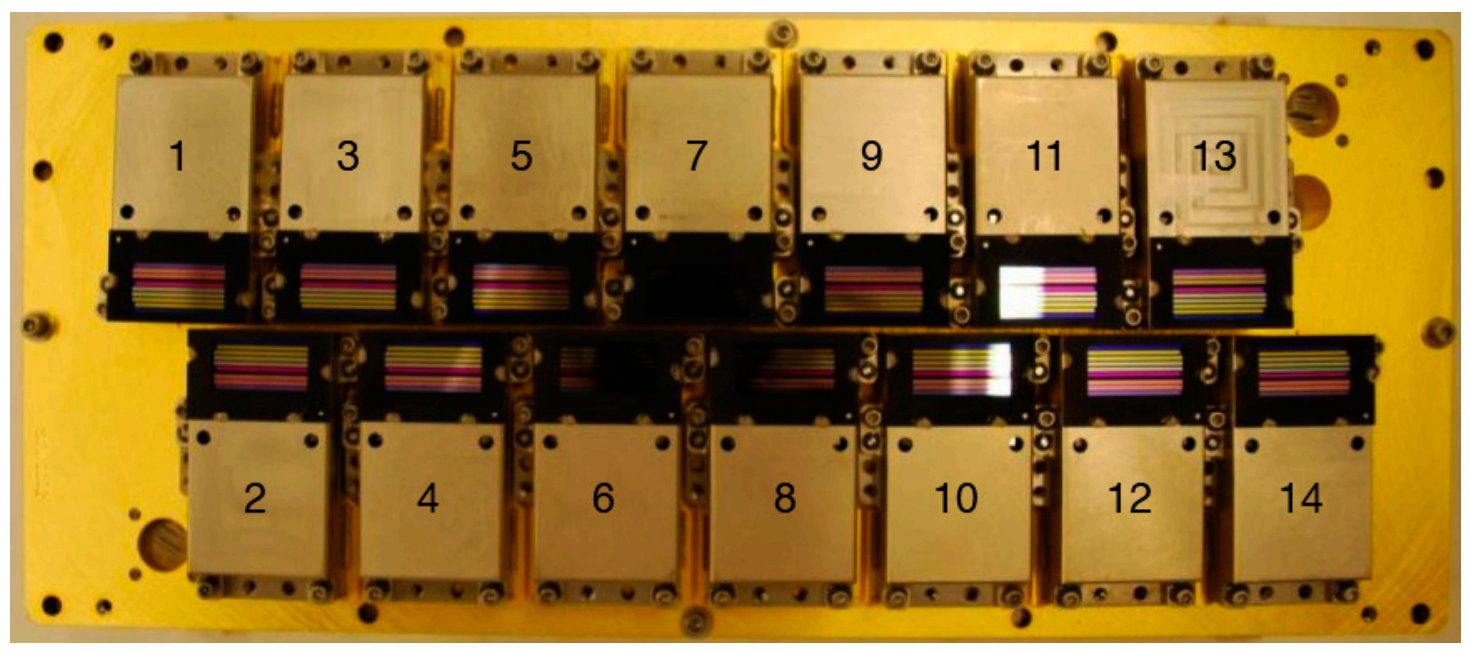

The filter assemblies consist of nine filter strips cut from larger filter wafers. To account for losses in the production and assembly process several filter wafers were manufactured for each spectral band. Across a wafer, the spectral response is fairly uniform; the differences between wafers are larger. In the Green and Red bands, all 14 of the filter strips came from a single wafer; in the Cirrus band, from three different wafers; in the rest of the bands, from two wafers. Table 2 lists the wafer source of each module's filter. 
Table 2. Filter wafer distribution across the OLI focal plane. Numbers indicate which wafer was the source of the filter applied to each module (colors are to aid in visibility).

\begin{tabular}{ccccccccccc}
\hline $\begin{array}{c}\text { OLI FPM } \\
\text { Number }\end{array}$ & CA & Blue & Green & Red & NIR & Cirrus & SWIR1 & SWIR2 & Pan \\
\hline 1 & 2 & 3 & 1 & 1 & 2 & 3 & 2 & 1 & 2 \\
2 & 1 & 1 & 1 & 1 & 1 & 2 & 1 & 1 & 1 \\
3 & 1 & 1 & 1 & 1 & 1 & 1 & 1 & 1 & 1 \\
4 & 1 & 1 & 1 & 1 & 1 & 1 & 1 & 1 & 1 \\
5 & 2 & 1 & 1 & 1 & 2 & 2 & 2 & 1 & 2 \\
6 & 1 & 1 & 1 & 1 & 1 & 2 & 2 & 1 & 1 \\
7 & 2 & 3 & 1 & 1 & 2 & 3 & 2 & 2 & 2 \\
8 & 1 & 1 & 1 & 1 & 1 & 1 & 1 & 1 & 1 \\
9 & 1 & 1 & 1 & 1 & 1 & 1 & 1 & 1 & 1 \\
10 & 1 & 1 & 1 & 1 & 2 & 2 & 2 & 1 & 2 \\
11 & 1 & 1 & 1 & 1 & 1 & 2 & 1 & 1 & 1 \\
12 & 1 & 1 & 1 & 1 & 1 & 2 & 1 & 1 & 1 \\
13 & 2 & 3 & 1 & 1 & 2 & 3 & 2 & 2 & 2 \\
14 & 1 & 1 & 1 & 1 & 1 & 1 & 1 & 1 & 1 \\
\hline
\end{tabular}

\section{Spectral Measurements}

\subsection{Component Level Measurements}

The spectral response of each component in the OLI optical path was measured independently: the transmission of each filter wafer, the reflectance of the four mirrors, the response of the detectors, and the transmission of the focal plane window. The relative responses of all of the components other than the spectral filters are shown in Figure 2.

Figure 2. Relative responses of OLI optical path components over entire spectral range of the instrument, including some out-of-band response. Detector relative response is radiance (power) based.

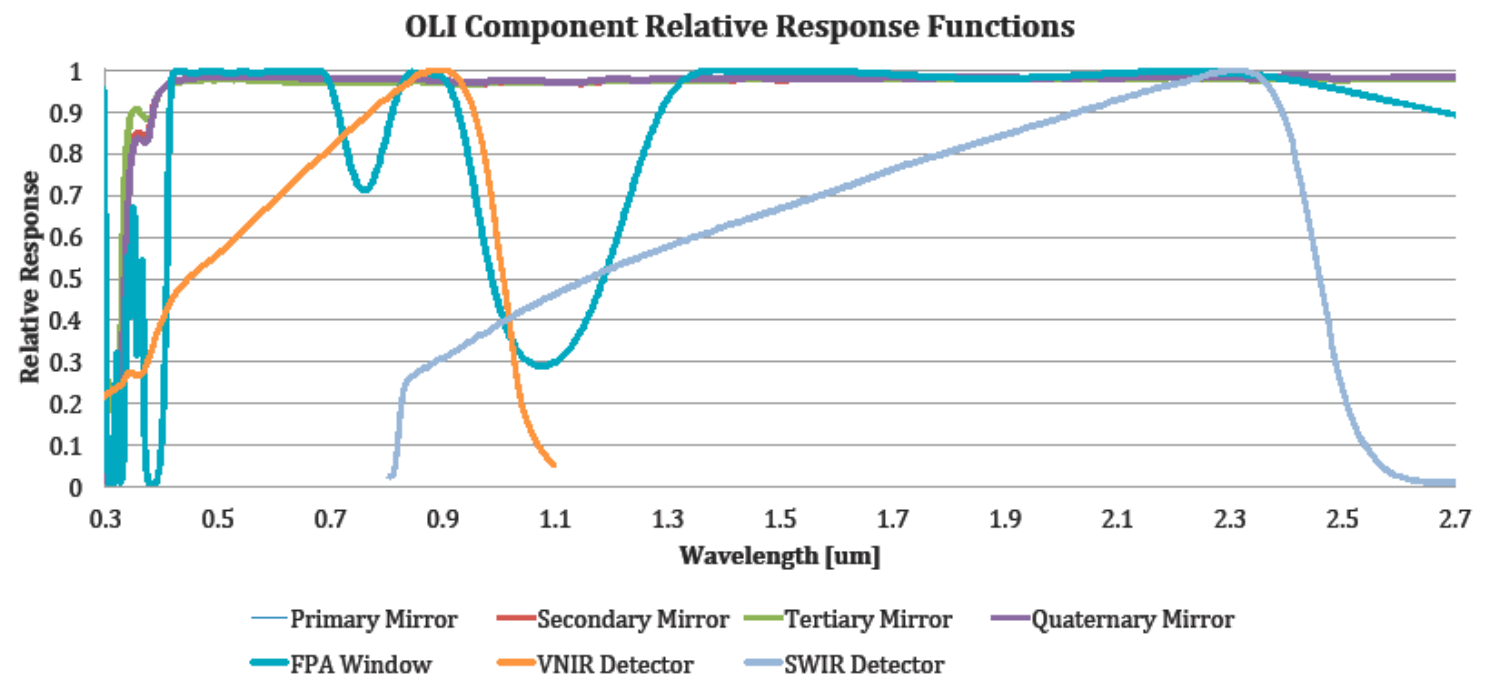


The spectral transmission of each filter wafer was measured at nine positions by the filter provider, Barr Associates (now part of Materion). Measurements were made at ambient temperature and with collimated light; models were used to predict the thermal and angular shifts when used in the instrument. The reflectance of each mirror's witness sample was measured at three positions by Ball Aerospace using a Cary 5000 spectrometer. Relative spectral responses of 6 VNIR witness detectors and 32 SWIR witness detectors were measured in vacuum at $210 \mathrm{~K}$, by the detector provider, Raytheon Vision Systems (RVS). The spectral transmission of a witness sample of the focal plane assembly window was measured at five positions by Sonoma Photonics. A system-level response for each module of each band was estimated by averaging all the measurements of each component and combining them (Figure 3). The measurements of each component covered the spectral range of 350-1100 nm for the VNIR bands and 800-2600 $\mathrm{nm}$ in the SWIR bands. While not a complete out-of-band assessment, this range allowed for the initial inspection of the out-of-band response (the response to radiance outside of the prescribed spectral wavelength range) (Figure 4).

Figure 3. Estimated system-level relative radiance spectral response function of each FPM of the OLI CA (upper left), Red (upper right), SWIR1 (lower left) and Cirrus (lower right) bands, bands which illustrate the wafer-to-wafer variability. All of the filters in the Red band originated from the same wafer. The filters in the CA and SWIR1 band come from two different wafers. The filters for the Cirrus band come from three different wafers.
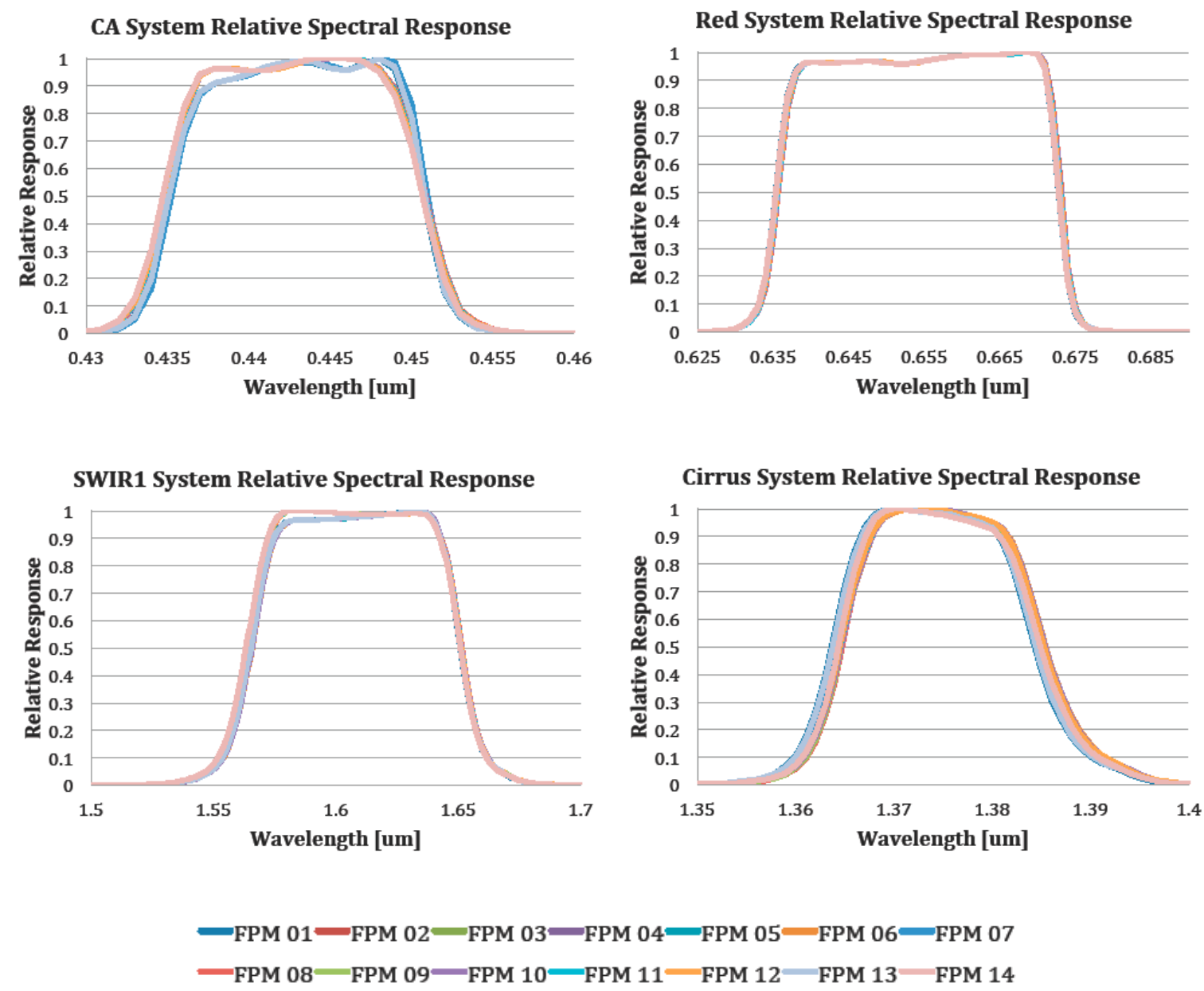
Figure 4. Estimated system-level relative radiance spectral response function of each FPM of the OLI CA (upper left), Red (upper right), SWIR1 (lower left) and Cirrus (lower right) bands including the out-of-band response. The out-of-band response is below 1e-3 in all FPMs of all bands.
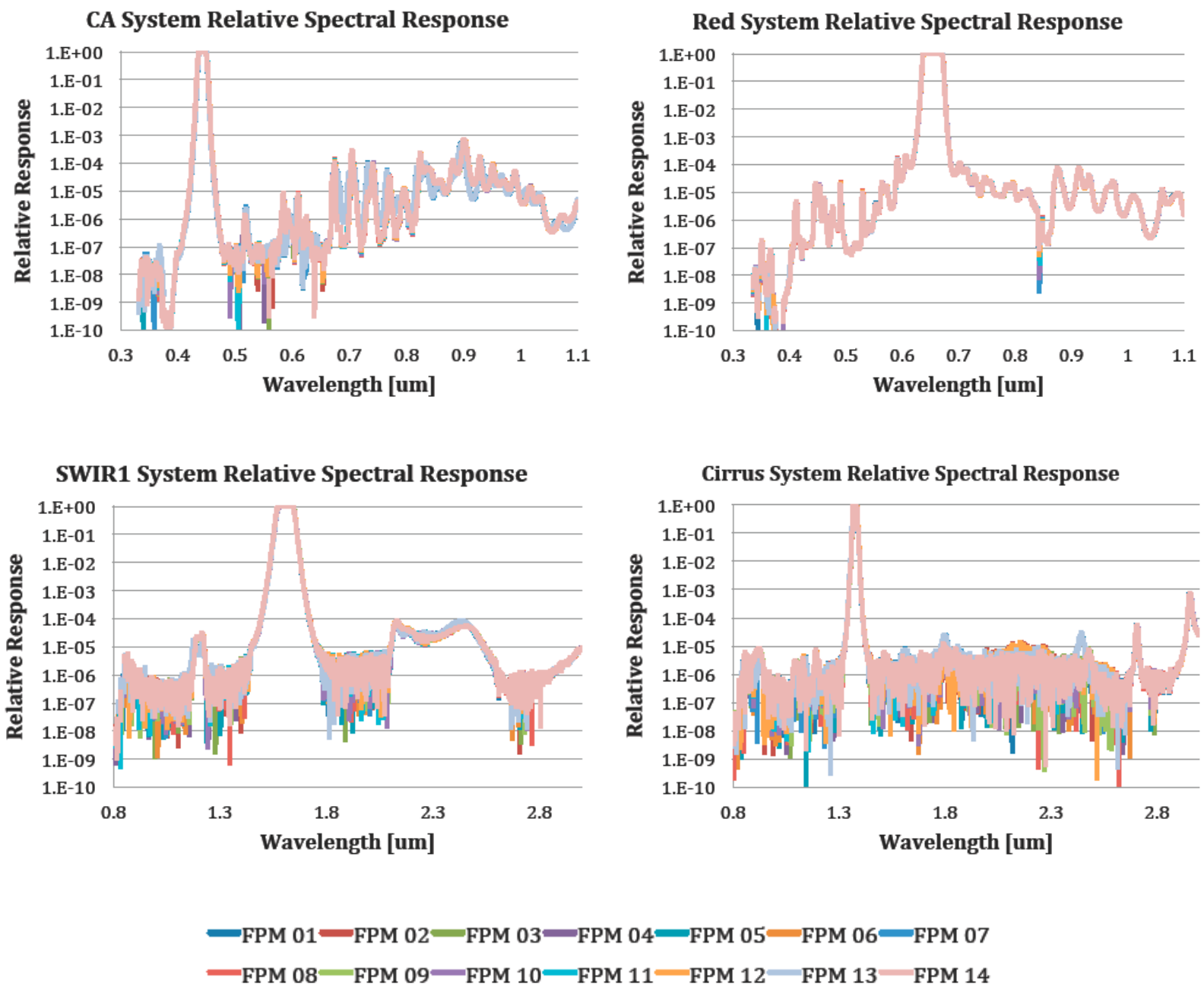

The predicted system-level response based on the component measurements indicated that the out of band response was low enough and that the spectral response across the focal plane was uniform enough to proceed with the development of the instrument.

\subsection{Out-of-Band Measurements}

The OLI out-of-band responses were measured at the FPM level, when the detectors and spectral filters were mated. At this level of integration, the optical components controlling the out-of-band response are measured directly and the smaller contributors, the telescope optics and focal plane window (Figure 2), can be analytically combined with little addition to the uncertainty. The telescope mirrors are highly reflective (total $>90 \%$ ) and essentially flat from 450-2500 nm and have almost no impact on the out-of-band response across that range. Below $450 \mathrm{~nm}$, the mirror reflectance drops off. The Focal Plane Array (FPA) window is flat and highly transmissive from 420-700 nm, 820-900 nm and $1300-2400 \mathrm{~nm}$. Its transmission is highly structured below $420 \mathrm{~nm}$. The window transmission 
combined with the mirrors' reflectance gives reduced sensitivity and the common structure to all bands below $420 \mathrm{~nm}$. The FPA window also contributes to the reduced response between 900 and $1300 \mathrm{~nm}$.

To measure the out-of-band response, each flight FPM was placed in an evacuated Dewar and cooled to the nominal operational temperature $(210 \mathrm{~K})$ (Figure 5). The FPM was illuminated through a window in the chamber with monochromatic light. A single monochromator with appropriate order sorting filters illuminated by a tungsten halogen lamp provided the light. The light exiting the monochromator was set at a distance to flood illuminate the FPM through a baffle and diffuser at the correct f-number. The VNIR bands were measured from $330-1100 \mathrm{~nm}$ at $10 \mathrm{~nm}$ increments (both sampling and bandwidth) for the out-of-band spectral regions. A calibrated silicon detector provided the reference for the monochromator output. The SWIR bands were measured from $800-2700 \mathrm{~nm}$ at $20 \mathrm{~nm}$ increments (both sampling and bandwidth) for the out-of-band regions. In-band responses were also measured at $2 \mathrm{~nm}$ increments for the VNIR and $4 \mathrm{~nm}$ increments for the SWIR. The in-band measurements allowed for normalization of the out-of-band response to the system level in-band response. Calibrated germanium and lead-sulfide detectors provided the references for the SWIR measurements. The resulting response for each detector was corrected for the transmission of the Dewar window and the variation in the monochromator output with wavelength and time using the reference detectors, and normalized to the peak in-band response. The responses were averaged across the full FPM and then across all 14 flight FPMs. To predict the full instrument out-of-band response, the FPM measurements were multiplied by the reflectance of the telescope mirrors and the transmission of the Focal Plane Window (Figure 2).

The band average out-of-band responses based on FPM-level measurements are shown in Figure 6. The data for the individual band average responses, the component level out-of-band predictions and the variability between the 14 FPMs are available in the spreadsheet posted at http://landsat.gsfc. nasa.gov/?p=8829.

Figure 5. Test setup for FPM-level out-of-band spectral testing. The module is inside an evacuated Dewar. A tungsten halogen lamp illuminates the slit of a monochromator. The monochromatic light is piped through a baffle and diffuser to the module.

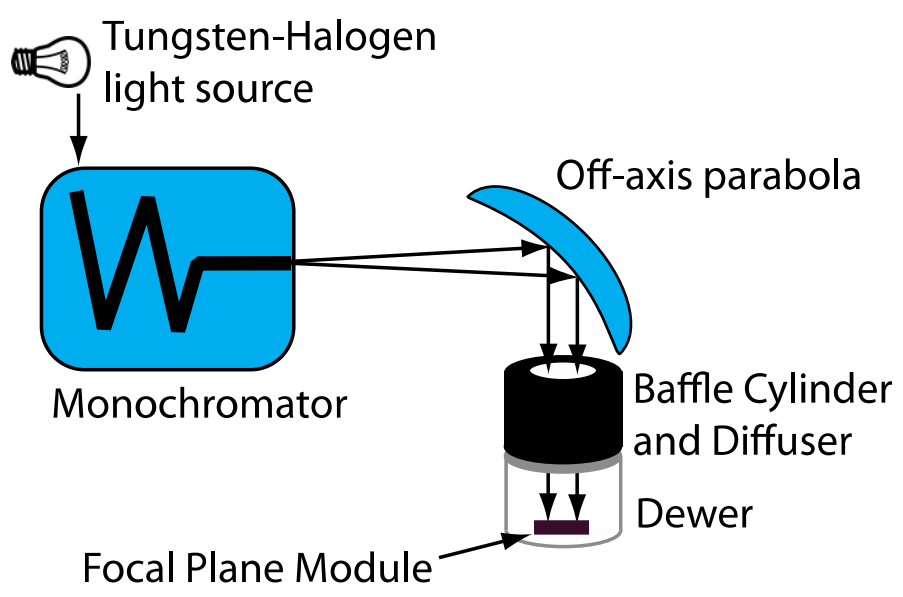

There are features in the data (Figure 6) that result from the test conditions as opposed to the instrument spectral response. These features are detailed below. In general, the FPM-level 
measurements should be considered an upper bound for the true out-of-band response and the component-level roll-up a lower bound (Figure 6).

Figure 6. (a) OLI VNIR bands and (b) SWIR bands out-of-band response based on FPM-level measurements with other optical components (mirrors and FPA window) included analytically. The plots are scaled to show the out-of-band response, so the in-band response is not shown here.

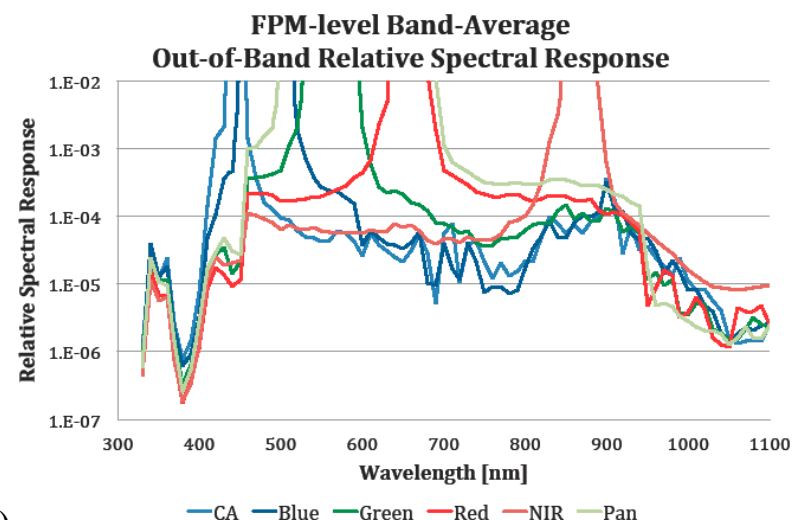

(a)

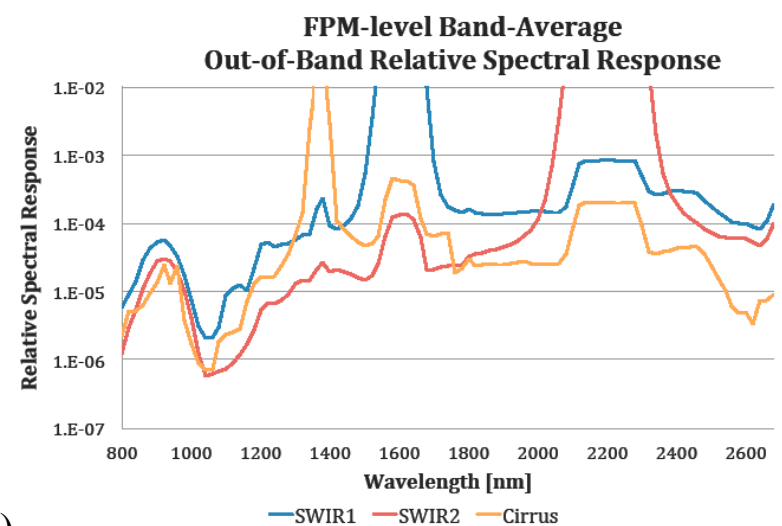

(b)

(1) Order-sorting filter effects: For the VNIR bands, the order-sorting filters were changed between 450 and $460 \mathrm{~nm}, 590$ and $600 \mathrm{~nm}$ and 940 and $950 \mathrm{~nm}$. The common discontinuities in the apparent out-of-band responses at these wavelengths are due to these filter changes, e.g., Green, Red, NIR and Pan between 450 and $460 \mathrm{~nm}$; CA and Blue between 590 and $600 \mathrm{~nm}$ and Green, Red and Pan between 940 and $950 \mathrm{~nm}$. The order-sorting filters inserted for measurements at $450 \mathrm{~nm}$ and below also blocked light above $500 \mathrm{~nm}$ (as well as below $250 \mathrm{~nm}$ ). This significantly reduced the apparent out-of-band response due to spectral stray light in the single monochromator set up for bands with the band pass above $500 \mathrm{~nm}$. Similarly, the order-sorting filter, inserted for measurements at $950 \mathrm{~nm}$ and above, filters out light below $705 \mathrm{~nm}$ causing bands with bandpasses below this wavelength to show reduced response (particularly green, red and pan). For the SWIR bands, the order sorting filter effects are not as visually evident, with the exception of between 1740 and $1760 \mathrm{~nm}$ in the Cirrus band.

(2) In-band to out-of-band measurement effects: As indicated, the in-band measurements were taken at a finer spectral resolution than the out-of-band ones. The in-band and out-of-band data sets are merged together at approximately the $1 \%$ response points. Due to the differences in the bandwidths, these measurements do not always merge smoothly, producing discontinuities in the apparent resulting response.

All three SWIR bands in Figure 6 show higher out-of-band response in the spectral ranges corresponding to the other two SWIR bands regions than in surrounding spectral regions. For example, the SWIR1 band response (Figure $7 \mathrm{~b}$ ) approaches $1 \mathrm{e}-3$ in the range corresponding to SWIR2, compared to about $1 \mathrm{e}^{-4}$ in surrounding regions. Additional pre-launch testing and analysis indicated that this was crosstalk between the bands, most likely optical crosstalk within the detector material. Subsequent post-launch imagery and analysis discussed later in this paper also showed the crosstalk.

For the OLI pushbroom architecture, there are significant differences in the impact of out-of-band response due to crosstalk as opposed to filter out-of-band response, when the sensor is viewing a non-uniform scene. This is because at any instant different bands are viewing different regions of the 
scene. Thus out-of-band response originating from crosstalk is both out-of-field as well as out-of-band, whereas out-of-band signal due to imperfect filter cutoffs is purely out-of-band.

Figure 7. FPM-average out-of-band response for FPM9 of (a) NIR and (b) SWIR1 bands. In general, the component-level measurements indicate less out-of-band response than the module test and it is difficult to know how much of this difference is attributable to the test setup and not OLI. However, in the SWIR bands there is a cross-talk feature that on-orbit analyses show is real. The increase in out-of-band response centered at $2200 \mathrm{~nm}$ corresponds to the SWIR2 band, indicating the presence of spectral crosstalk.

(a)

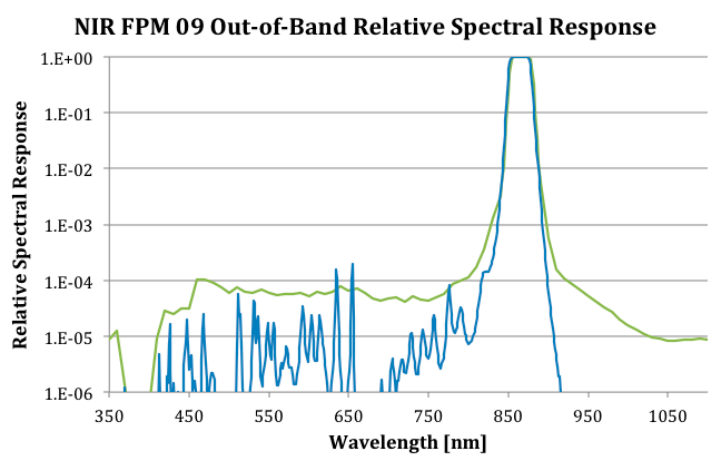

—Module-level response -Component-level system reponse

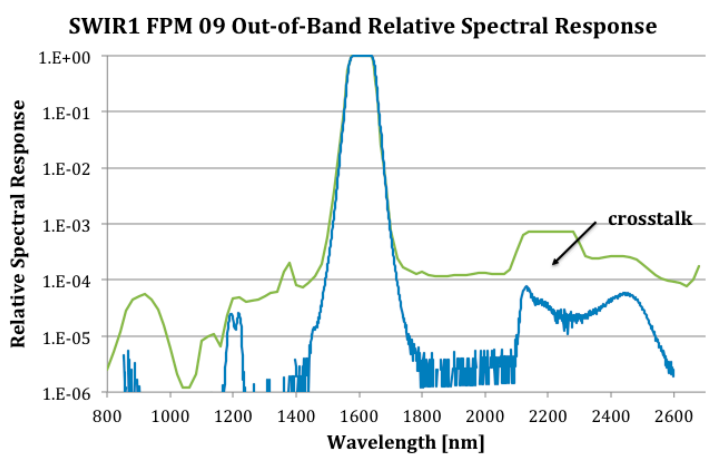

(b)

\subsection{Instrument Level Measurements}

After the OLI was assembled, it was characterized in a thermal vacuum chamber. The spectral characterization was performed using a double monochromator located outside the chamber (Figure 8a). A tungsten halogen lamp illuminated the input slit of a double monochromator. At the output slit of the monochromator a beam splitter sent part of the beam to a monitor detector and part through a collimator and a window in the thermal vacuum chamber to the OLI (Figure 8b). The OLI was pointed using ground support equipment so that the collimated beam covered 16 different locations for each band, one position at the center of each FPM and one location each at the ends of the two extreme cross-field FPMs. The size of the beam was such that there was sufficient signal to characterize approximately 60 detectors at each location. At each location that OLI data were collected, the monochromator stepped through the OLI spectral bandpass at the wavelength intervals given in Table 3 . The characterization was performed across a fixed wavelength range for each band that was designed to achieve responses down to at least the 0.005 relative spectral response point. Each OLI detector's digital response was offset corrected, normalized for temporally and spectrally dependent variations in the illuminating radiance, and adjusted for the transmission of the path optics:

$$
\beta^{\prime}(\lambda)=\frac{\left(Q(\lambda)-Q_{0}\right) * R_{m}(\lambda)}{\tau_{\text {OLIpath }}(\lambda)}
$$

where $\beta^{\prime}(\lambda)$ is the derived spectral response, $Q(\lambda)$ is the digital response of an OLI detector to the monochromator signal for the specified wavelength, $Q_{0}$ is the digital response of an OLI detector to no input radiance, $R_{m}(\lambda)$ is the wavelength dependent correction factor for the radiance output of the monochromator based on the monitor output and the monitor's radiometric calibration and $\tau_{\text {OLIpath }}(\lambda)$ is 
the transmission of the optical path between the beam splitter and the OLI. The spectral response is then normalized to unity at the peak response:

$$
\beta(\lambda)=\frac{\beta^{\prime}(\lambda)}{\beta^{\prime}(\lambda)_{\max }}
$$

where $\beta^{\prime}(\lambda)_{\max }$ is the maximum value of the spectral response $\beta^{\prime}(\lambda)$.

Figure 8. Test setup for OLI instrument level spectral testing; (a) The OLI is inside a thermal vacuum chamber with the entrance aperture aligned with the window on the chamber. The OLI detectors are looking at the output of a double monochromator, one module at a time; (b) A tungsten halogen lamp illuminated the input slit of a double monochromator. At the output slit of the monochromator a beam splitter sent part of the light to a monitor detector and part through a collimator and a window in the thermal vacuum chamber to the OLI.

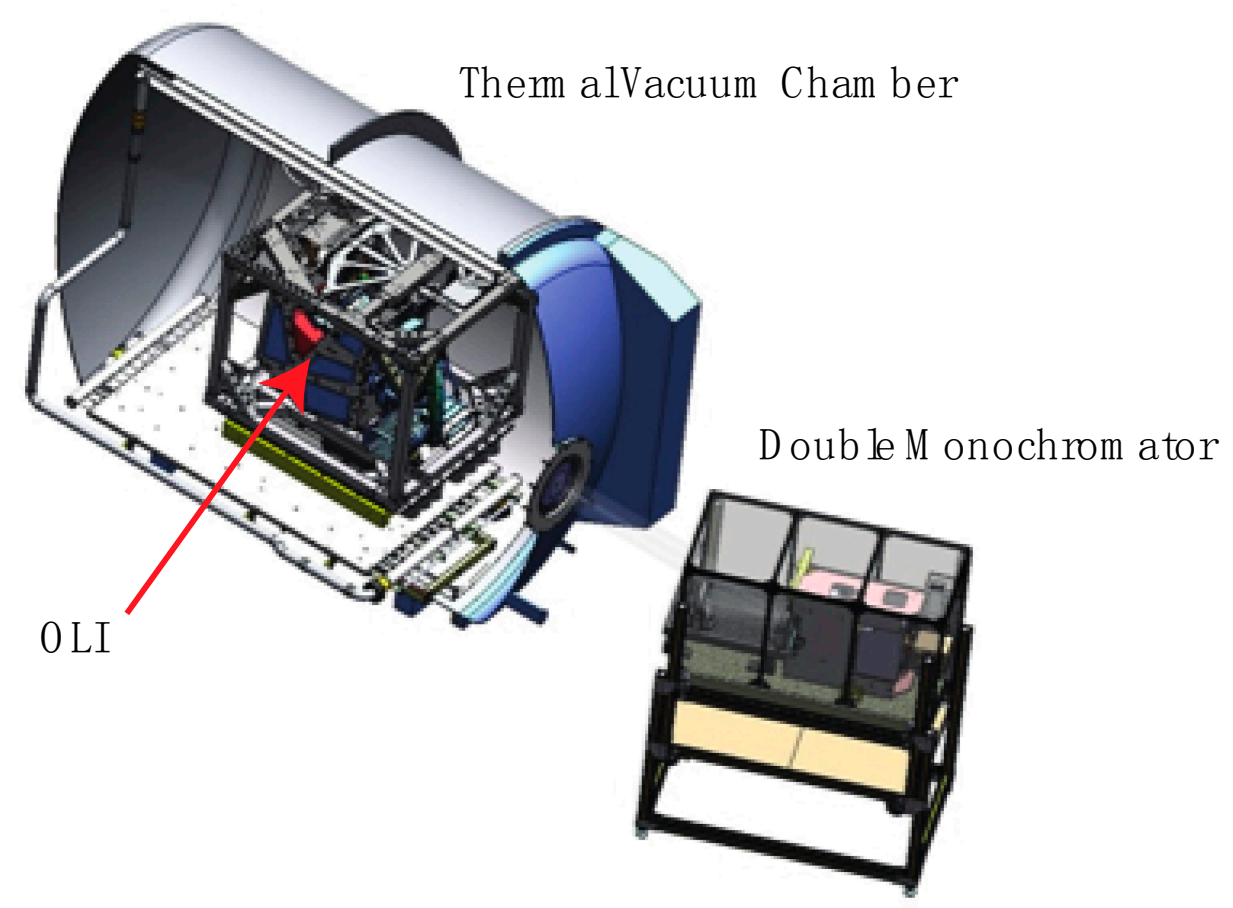

(a)

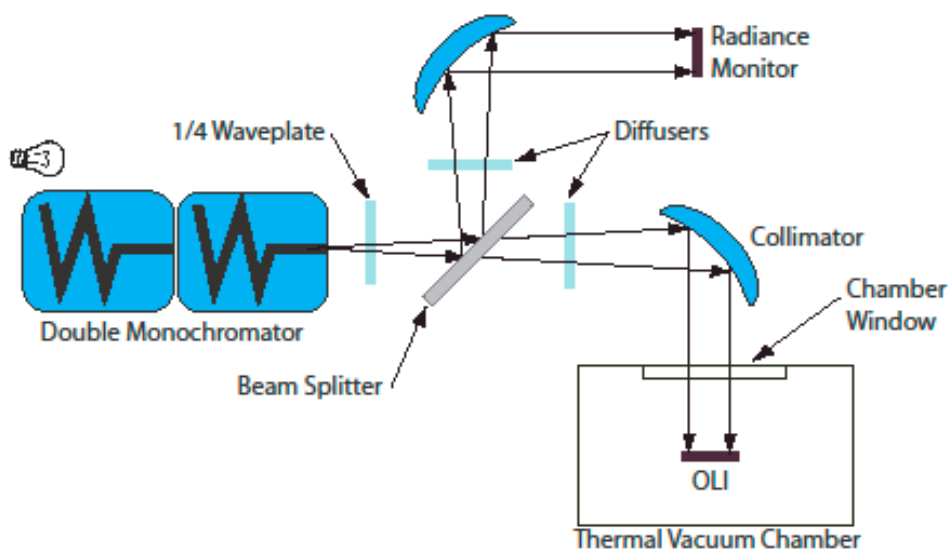

(b) 
Table 3. Sampling specifications during pre-launch spectral testing of OLI.

\begin{tabular}{cccc}
\hline $\begin{array}{c}\text { OLI Spectral } \\
\text { Band }\end{array}$ & $\begin{array}{c}\text { Monochromator } \\
\text { Sampling } \\
\text { Bandpass (nm) }\end{array}$ & $\begin{array}{c}\text { Monochromator } \\
\text { Step Size (nm) }\end{array}$ & $\begin{array}{c}\text { Monochromator } \\
\text { Bandpass (nm) }\end{array}$ \\
\hline CA & $427-459$ & 1 & 1 \\
Blue & $436-528$ & 1 & 1 \\
Green & $513-611$ & 1 & 1 \\
Red & $626-692$ & 1 & 1 \\
NIR & $830-901$ & 1 & 1 \\
SWIR1 & $1516-1699$ & 2 & 2 \\
SWIR2 & $2038-2356$ & 2 & 2 \\
Pan & $488-693$ & 1 & 1 \\
Cirrus & $1341-1410$ & 2 & \\
\hline
\end{tabular}

The FPM-average relative spectral responses of sample bands are shown in Figure 9, along with the uncertainty in the measurement. Repeatability measurements were made on the center 60 detectors of a single module and used to estimate uncertainty of the response. The differences between the FPMs' responses are primarily due to the spectral differences between the source wafers. The Red band is shown as an illustration of a band where all the filter sticks were cut from the same filter wafer while the CA filters come from two wafers.

During this test, estimates of the in-band spectral characteristics were made: spectral band edges, center wavelength, average response, minimum response, and bandpass uniformity (Table 4). The bandpass uniformity is the difference between the per-detector full-width, half-maximum band edges. A sample of the bandpass uniformities is shown in Figure 10.

The band average results of this instrument level test are being provided as the official relative spectral response (RSR) of OLI. These are available on the Landsat-8 web site at http://landsat. gsfc.nasa.gov/?p=5779.

Table 4. Summary of band-average spectral response bandwidths and edges as determined from the full-width, half-maximum for each band.

\begin{tabular}{cccccc}
\hline Band \# & Band & $\begin{array}{c}\text { Center } \\
\text { Wavelength (nm) }\end{array}$ & $\begin{array}{c}\text { Bandwidth } \\
(\mathbf{n m})\end{array}$ & $\begin{array}{c}\text { Lower Band } \\
\text { Edge (nm) }\end{array}$ & $\begin{array}{c}\text { Upper Band } \\
\text { Edge (nm) }\end{array}$ \\
\hline 1 & CA & 443.0 & 16.0 & 435.0 & 451.0 \\
2 & Blue & 482.0 & 60.0 & 452.0 & 512.1 \\
3 & Green & 561.4 & 57.3 & 532.7 & 590.1 \\
4 & Red & 654.6 & 37.5 & 635.9 & 673.3 \\
5 & NIR & 864.7 & 28.3 & 850.5 & 878.8 \\
6 & SWIR1 & 1608.9 & 84.7 & 1566.5 & 1651.2 \\
7 & SWIR2 & 2200.7 & 186.7 & 2107.4 & 2294.1 \\
8 & Pan & 589.5 & 172.4 & 503.3 & 675.7 \\
9 & Cirrus & 1373.4 & 20.4 & 1363.2 & 1383.6 \\
\hline
\end{tabular}


Figure 9. (a) OLI system FPM-average spectral response function for all modules on the focal plane for sample bands. (b) Uncertainty in response function based on the repeatability test, where the same test was repeated six times. The uncertainty is the standard error over all six tests and all 60 illuminated detectors.
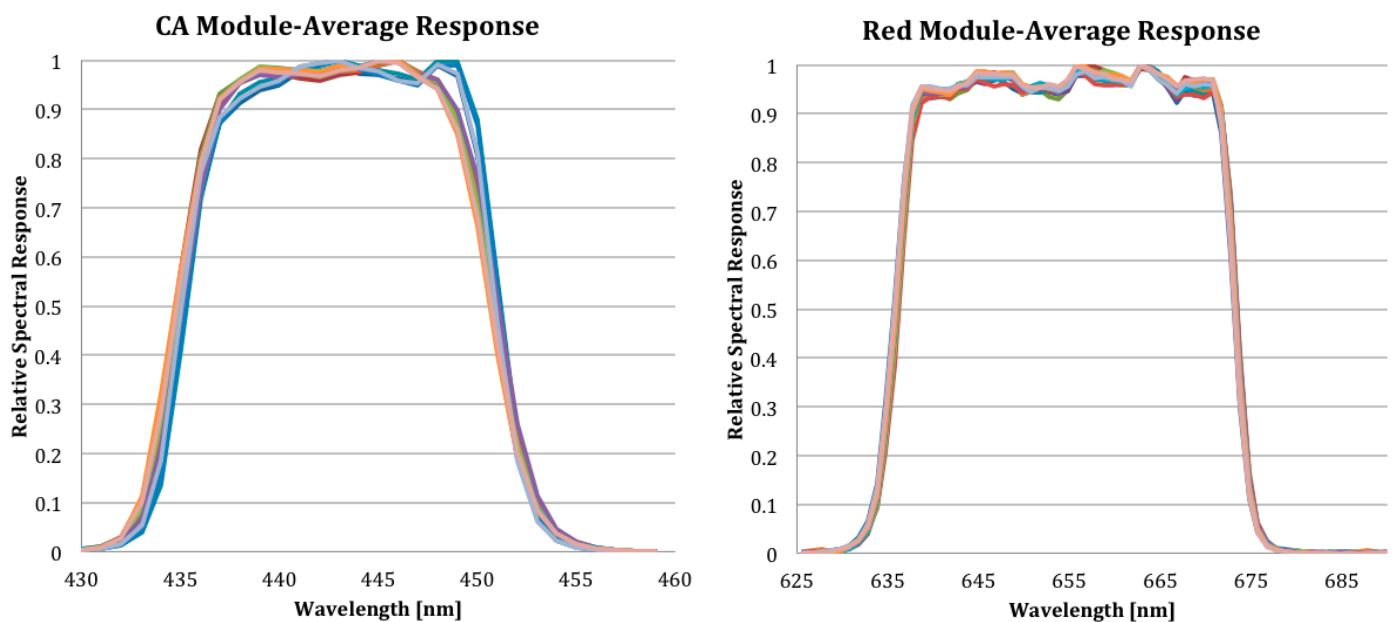

(a)
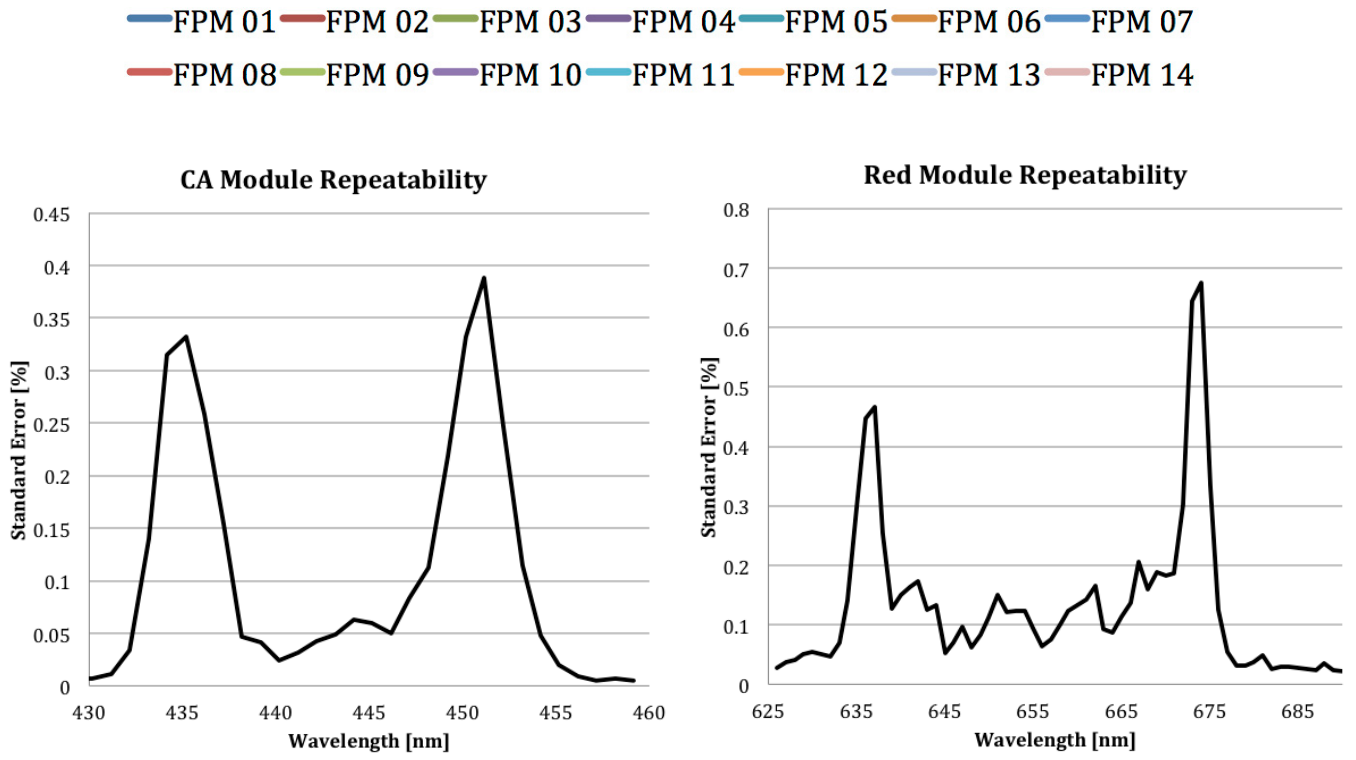

Figure 10. The spectral bandpass uniformity for the CA (left) and Red (right) bands, as measured by the variation in the bandpass over all detectors tested during the instrument level tests. The uniformity is relative to the median bandpass.

CA Spectral Bandpass Uniformity

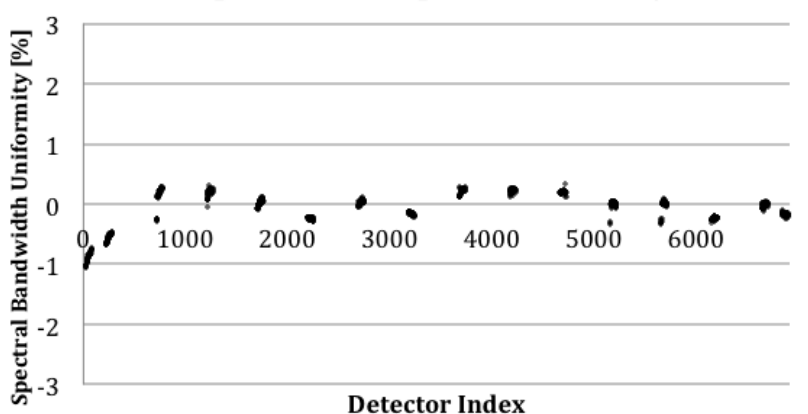

Red Spectral Bandpass Uniformity

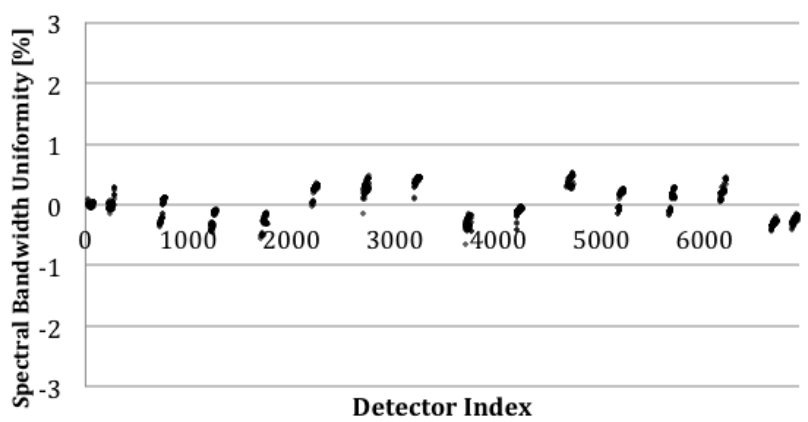




\section{Spectral Uniformity}

Given the pushbroom architecture of the OLI, the variation in spectral response between pixels results in radiometric differences across the focal plane that appears as streaks (detector-to-detector variations) or bands (FPM-to-FPM variations) in the along-track direction. These differences cannot be readily calibrated out, as they are target dependent. On-orbit, the solar diffuser, which has a different spectral radiance than any Earth spectrum, is used to flat-field the data. A simulation was performed to determine the amount of residual spectrally-related variability. This difference is included in the overall radiometric uncertainty [4].

The differences in RSR between modules will result in different variations in integrated radiance across a spatially uniform scene for targets with different reflectance spectra. This effect is simulated for two sample targets types, vegetation and bare soil. The spectral radiance in each band (b) for each FPM (f) for each target $(\mathrm{t}), L_{\lambda}(b, f, t)$ is calculated using the instrument-level RSR (except the Cirrus band):

$$
L_{\lambda}(b, f, t)=\frac{\int L_{\lambda}(t, \lambda) * \beta(b, f, \lambda) d \lambda}{\int \beta(b, f, \lambda) d \lambda}
$$

where $L_{\lambda}(t, \lambda)$ is the target top-of-atmosphere spectral radiance (Figure 11) and $\beta(b, f, \lambda)$ is the average relative spectral response for each FPM. To simulate the effect of flat-fielding the data using the solar diffuser, Equation (3) is also used to calculate the solar radiance in each FPM, $L_{\lambda}(b, f$, sun), in each band and the average solar radiance across all FPM's, $\bar{L}_{\lambda}$ (b, sun), and used to normalize the responses per Equation (4).

$$
L_{\lambda, n}(b, f, t)=\frac{L_{\lambda}(b, f, t) * \bar{L}_{\lambda}(b, \text { sun })}{L_{\lambda}(b, f, \text { sun })}
$$

The percentage differences between the band-average and per-FPM normalized radiances, $L_{\lambda, n}(b, f, t)$ for the sample targets are plotted in Figure 12 for two bands and the maximum and average discontinuities between adjacent FPMs are given in Table 5. In all bands, there are a few tenths of percent difference between FPMs due to the RSR differences. In addition, the RMS variability introduced across the scene due to spectral variation for these two targets is less than $0.1 \%$ (Table 5).

Figure 11. Top-of-atmosphere radiances for two surface types for OLI to use as sample targets in simulations.

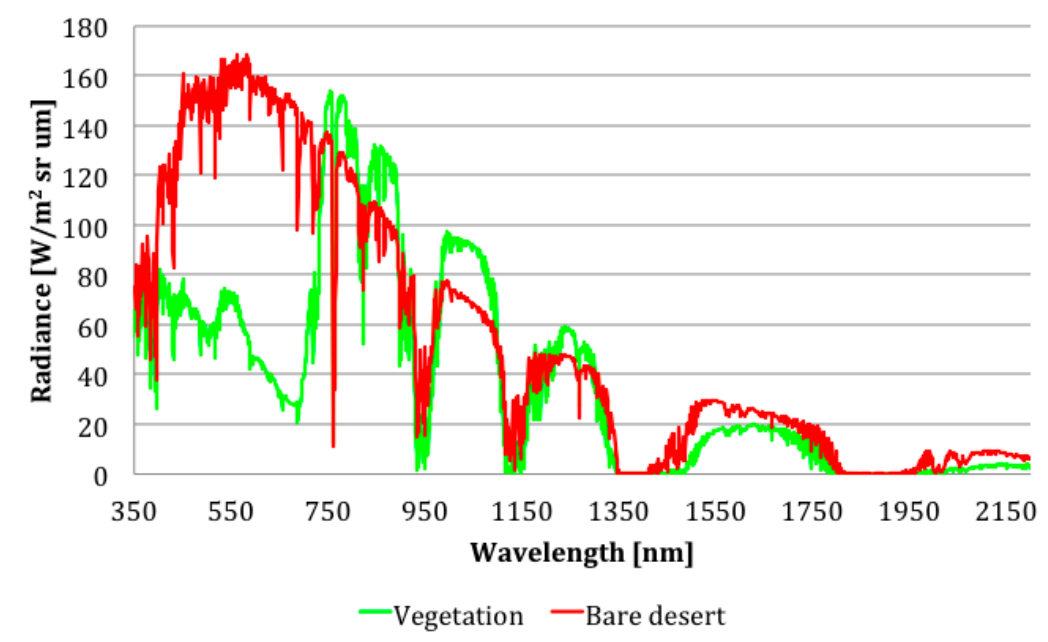


Figure 12. Radiance differences strictly due to the spectral response differences in FPMs for the sample targets, calculated using a band-average RSR, for the CA (left) and Red (right) bands. There is no difference in the solar data due to differences in the RSR because the solar radiances are used to flat-field the data.
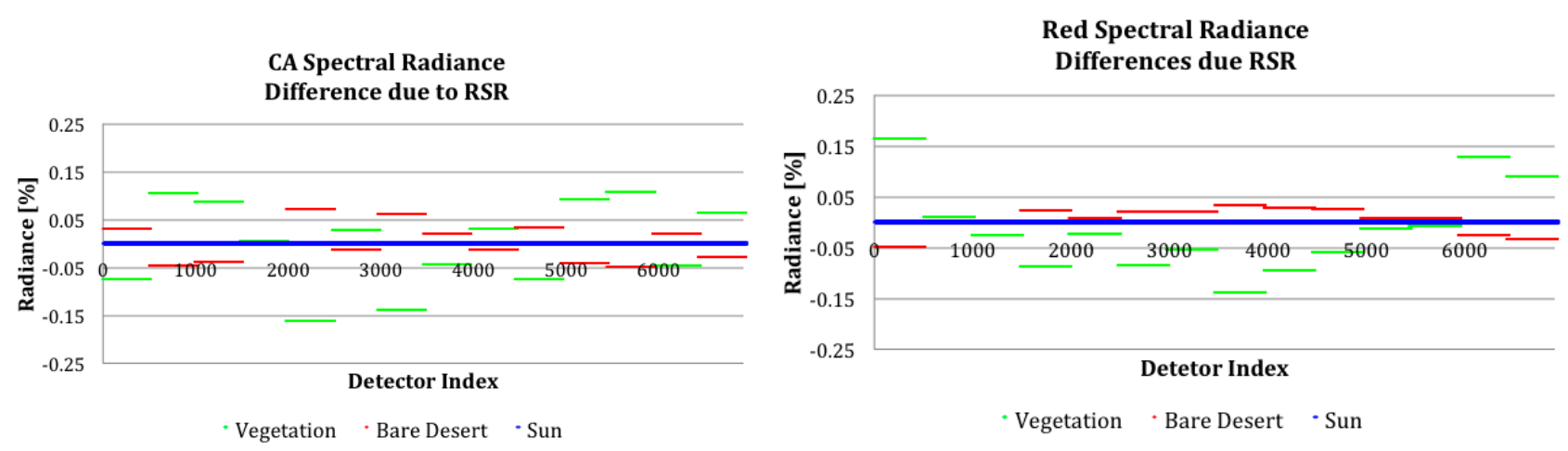

Table 5. The maximum and average radiance differences between adjacent FPMs across the focal plane along with the RMS variability due strictly to the spectral response differences in FPMs for sample targets calculated using a band average RSR. The vegetation discontinuities are larger than the soil, likely due to the fact that the soil is more spectrally similar to the solar spectra and solar data are used to flat-field the results. The Cirrus band is not included here because the signal in this band is so weak.

\begin{tabular}{c|c|c|c|c|c|c}
\hline & \multicolumn{2}{|c|}{ Maximum Discontinuity } & \multicolumn{2}{c|}{ Average Discontinuity } & \multicolumn{2}{c}{ RMS Variability } \\
\hline Band & Vegetation (\%) & Soil (\%) & Vegetation (\%) & Soil & Vegetation (\%) & Soil (\%) \\
\hline CA & 0.19 & 0.08 & 0.12 & 0.05 & 0.09 & 0.04 \\
Blue & 0.16 & 0.03 & 0.05 & 0.01 & 0.07 & 0.02 \\
Green & 0.11 & 0.02 & 0.05 & 0.01 & 0.07 & 0.01 \\
Red & 0.15 & 0.05 & 0.06 & 0.01 & 0.09 & 0.02 \\
NIR & 0.11 & 0.02 & 0.05 & 0.01 & 0.04 & 0.01 \\
SWIR1 & 0.16 & 0.03 & 0.10 & 0.08 & 0.09 & 0.01 \\
SWIR2 & 0.07 & 0.35 & 0.03 & 0.08 & 0.03 & 0.08 \\
Pan & 0.19 & 0.05 & 0.08 & 0.02 & 0.05 & 0.02 \\
\hline
\end{tabular}

\section{On-Orbit Spectral Response Observations}

There is no way to readily validate or monitor most aspects of the on-orbit OLI spectral response. If a change in the spectral response of the OLI is suspected, the best that can be done is to compare the changes in response to the various on-board calibrators, which have different spectral characteristics, to see if a spectral change can explain the response differences to the calibrator observations. No spectral changes have been suspected as yet, so no such analysis has been attempted.

However, there are some weak artifacts visible in the Cirrus band images on-orbit when there is a very low Cirrus signal. At least some of these artifacts now appear to be spectral crosstalk related, so they are discussed here. 


\subsection{On-Orbit Evidence of Spectral Crosstalk}

The Cirrus band is unique on OLI in that in many cases the in-band signal is very weak when there are strong VNIR and SWIR in-band signals. The Cirrus signal, when present, most often comes from clouds that appear bright on the dark atmosphere background. As such, it is the band where out-of-band response is likely to be most apparent, i.e., the radiance in the out-of-band regions is often much higher than the in-band radiance. Prelaunch measurements indicated some spectral crosstalk (Figure 6) and early on-orbit observations revealed that the Earth surface was sometimes weakly visible in the Cirrus band where it was not expected, i.e., under moist atmospheric conditions.

Closer investigation revealed that this surface visible in the Cirrus band was often misaligned from where the surface, if present, would occur in the Cirrus band (Figure 13). In particular, at land-water boundaries (Figure 13a), the odd and even FPMs in the geometrically corrected Cirrus band appear to be misaligned. Recognizing that this signal could be crosstalk, the Cirrus band images were reprocessed and treated geometrically as if the signal was coming from the location of the SWIR1 band. As shown in Figure 13b, the alignment was corrected. This is clear evidence that a good portion of Cirrus band signal over the land in this case is crosstalk from the SWIR1 band. The profiles show that the land-water contrast is about $0.1 \mathrm{~W} / \mathrm{m}^{2}$ sr $\mu \mathrm{m}$ in the Cirrus band and about $31 \mathrm{~W} / \mathrm{m}^{2} \mathrm{sr} \mu \mathrm{m}$ in the SWIR1 band. When converted to reflectance units, the effect is that about $0.2 \%$ of the SWIR 1 signal leaks into the Cirrus band, which is roughly consistent with the pre-launch estimates of Cirrus out-of-band response shown in Figure 6.

Observations of Cirrus images in very dry atmospheres indicate a surface signal that is properly aligned (Figure 14) and is therefore in-band. In Figure 14, the ice is approximately $1.4 \mathrm{~W} / \mathrm{m}^{2} \mathrm{sr} \mu \mathrm{m}$ and the island is approximately $0.4 \mathrm{~W} / \mathrm{m}^{2} \mathrm{sr} \mu \mathrm{m}$ at the top of the atmosphere, both brighter than the water and land $\left(0.05\right.$ and $0.15 \mathrm{~W} / \mathrm{m}^{2} \mathrm{sr} \mu \mathrm{m}$, respectively) in Figure 13 , when the crosstalk was apparent.

Figure 13. (a) Subset of a geometrically corrected Cirrus band image over the coast of Northern Africa showing odd and even FPM misalignment, with the associated along track profile (along the vertical red line in the image subset); (b) Same Cirrus image as (a) but geometrically corrected as if it were the SWIR1 band; and (c) SWIR1 band image of the same region and associated along track profile. Profiles are in units of $\mathrm{W} / \mathrm{m}^{2} \mathrm{sr} \mu \mathrm{m}$.

(a)

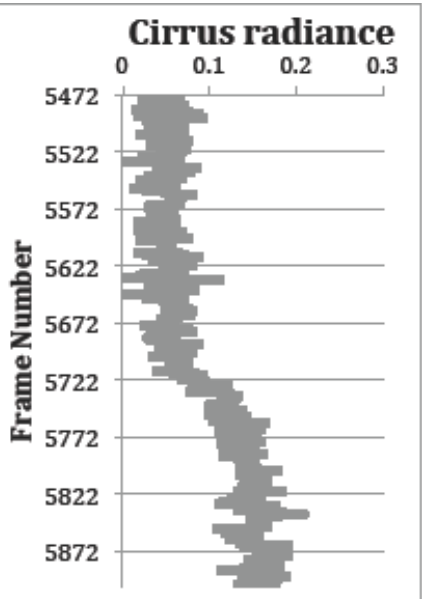


Figure 13. Cont.

(b)

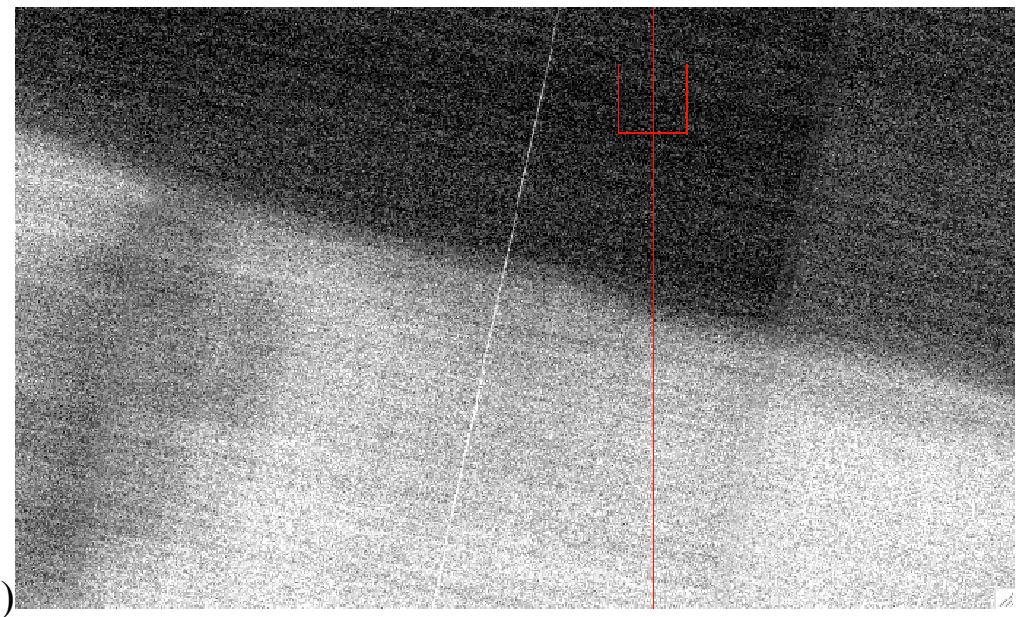

(c)
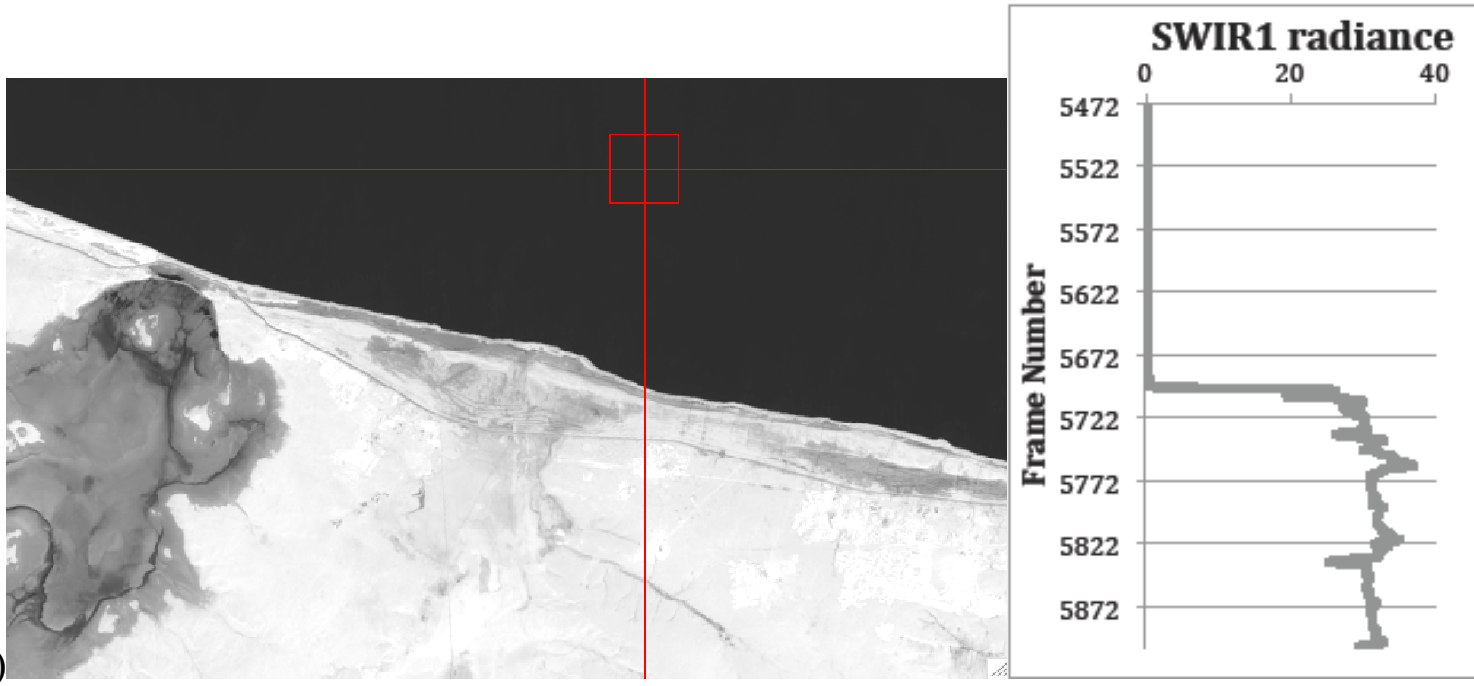

Figure 14. Cirrus band image in Northern Quebec of a frozen reservoir and island. The image was processed such that the FPM edges were not cropped, so the FPM boundary is apparent. The coastline of the island is properly registered across that boundary indicating that the Cirrus band imagery will be aligned when the signal is strong enough to make the crosstalk insignificant.

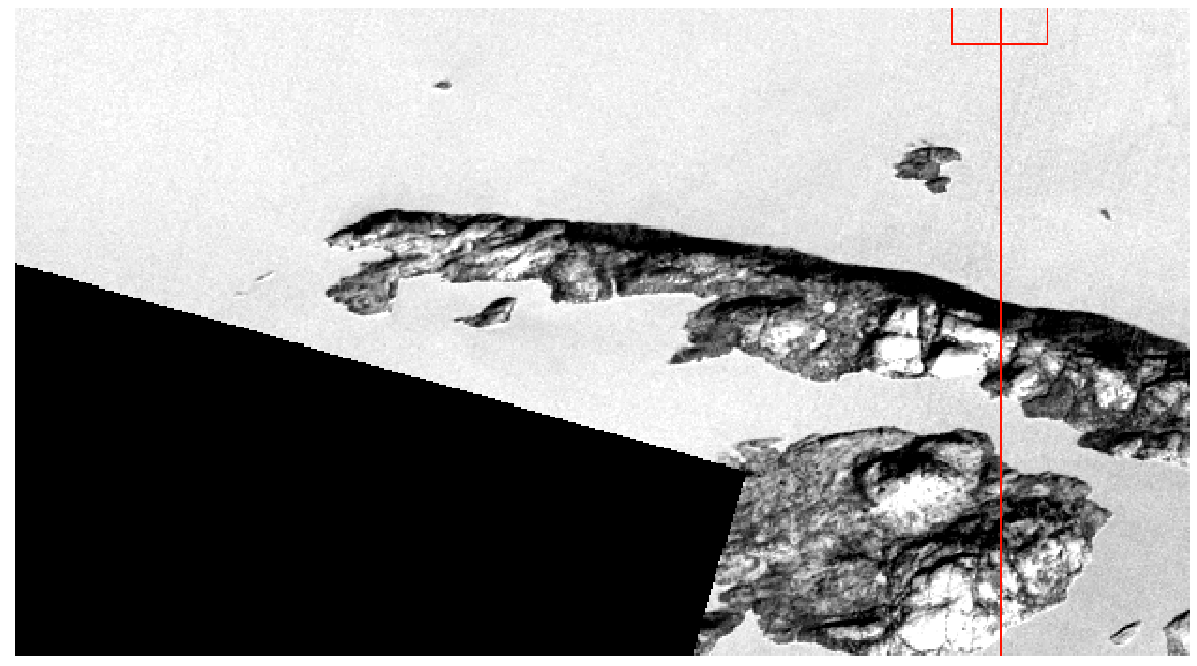




\subsection{Simulation of Out-of-Band Response Contribution}

In order to confirm that the pre-launch measured spectral response is consistent with the observed on-orbit crosstalk and to look for other cases of potential crosstalk that may not be readily visible in the imagery, simulations were performed using MODTRAN [5]. The full out-of-band RSR was derived from the FPM-level measurements combined with the OLI optical component measurements (Figure 4). A selection of atmospheres with a range of water content was processed through MODTRAN Version 4.1.1 (Table 6); three were standard atmospheres, the fourth was based on meteorological reanalysis data. A cirrus cloud was added within MODTRAN to one of the standard atmospheres, in order to quantify the effect of cirrus in the atmosphere. Four different surface targets out of the MODTRAN spectral library were run through each of the atmospheres (Figure 15). Most other MODTRAN options were set to the default except for the solar zenith angle, which was $45^{\circ}$ in all cases. Integrated spectral radiances for the Cirrus band were calculated based on the MODTRAN output:

$$
L_{\lambda}=\frac{\int_{\lambda=\lambda 1}^{\lambda=\lambda 2} L(\lambda) * \beta(\lambda) d \lambda}{\int_{\lambda=1000}^{\lambda=3000} \beta(\lambda) d \lambda}
$$

where $L(\lambda)$ is top-of-atmosphere spectral radiance as calculated by MODTRAN and $\beta(\lambda)$ is the relative spectral response which includes the out-of-band response. The limits for the wavelength ranges ( $\lambda 1$ and $\lambda 2$ ) are given in Table 7 . Spectral radiances were calculated over specific wavelength ranges to test the contributions of specific spectral regions to the total radiance (Table 7). The total radiance includes signal from the entire range of the RSR. The in-band radiance $\left(\mathrm{L}_{\lambda \text {,in-band }}\right)$ is only radiance from within the Cirrus band, as defined by the $0.1 \%$ points of the RSR. The in-band radiance includes contributions from the surface ( $\left.\mathrm{L}_{\lambda \text {,ground }}\right)$ and the atmospheric path $\left(\mathrm{L}_{\lambda \text {,path }}\right)$; the two are provided separately from MODTRAN:

$$
L_{\lambda, \text { in-band }}=L_{\lambda, \text { ground }}+L_{\lambda, \text { path }}
$$

The SWIR crosstalk radiances $\left(\mathrm{L}_{\lambda, \mathrm{SWIR} 1}\right.$ and $\mathrm{L}_{\lambda, \mathrm{SWIR} 2}$ ) were calculated with $\mathrm{SWIR}$ in-band wavelength ranges (Table 7) and the remaining out-of-band radiance $\left(\mathrm{L}_{\lambda, \mathrm{OOB}}\right)$ is calculated from the other contributions.

$$
L_{\lambda, O O B}=L_{\lambda, \text { total }}-L_{\lambda, \text { in-band }}-L_{\lambda, S W I R 1}-L_{\lambda, S W I R 2}
$$

Figure 15. Spectral reflectance of the four surface targets used in the out-of-band response analysis. The spectra originate from MODTRAN's spectral library [5].

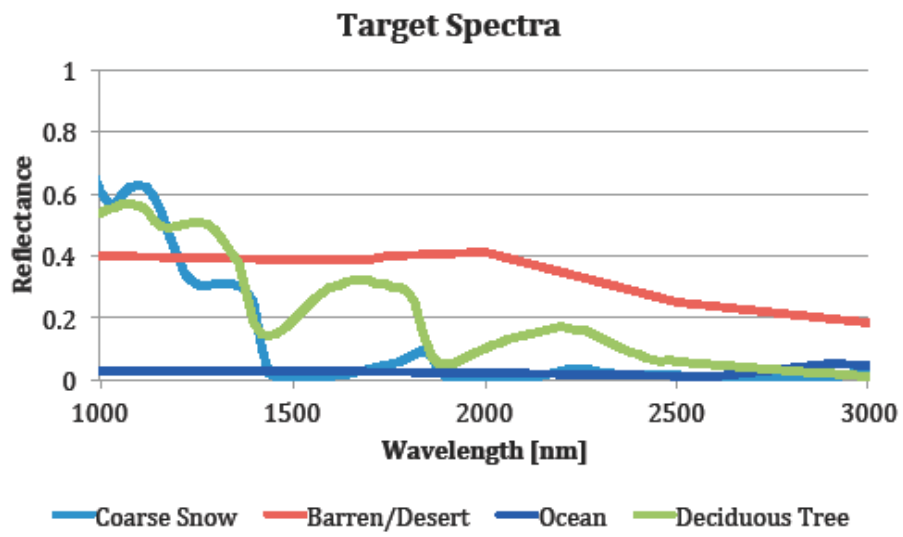


Table 6. Atmospheres used in the out-of-band response simulations.

\begin{tabular}{ccc}
\hline Atmosphere (Abbreviation) & Water Content $\mathbf{( g / \mathbf { c m } ^ { 2 } )}$ & Source \\
\hline Mid-Latitude Summer (ML-Sum) & 2.92 & MODTRAN \\
Mid-Latitude Winter (ML-Win) & 0.85 & MODTRAN \\
Greenland-September (Gnland) & 0.2 & NCEP Reanalysis (10 September 2013) \\
1976 US Standard + default 10 km Cirrus & 1.42 & MODTRAN \\
\hline
\end{tabular}

Table 7. Wavelength ranges for radiance components in spectral crosstalk analysis for the Cirrus band. The in-band ranges for all three bands were determined from the $0.1 \%$ response points of each band's response curve.

\begin{tabular}{cc}
\hline Radiance Component & $\begin{array}{c}\text { Wavelength Range } \\
\boldsymbol{\lambda} \mathbf{1}-\boldsymbol{\lambda} \mathbf{2}(\mathbf{n m})\end{array}$ \\
\hline Total & $1000-3000$ \\
\hline In-band $(0.1 \%$ threshold) & $1351-1391$ \\
\hline SWIR1 crosstalk & $1522-1681$ \\
\hline SWIR2 crosstalk & $2065-2331$ \\
\hline OOB (other than SWIR1, & $1000-1350$, \\
SWIR2) & $1392-1521$, \\
& $1682-2064$, \\
& $2332-3000$ \\
\hline
\end{tabular}

The spectral radiances for the components contributing to the total signal are shown in Figure 16. The simulation results were generally consistent with the on-orbit observations. In typical, cirrus-free atmospheres, as in both of the mid-latitude cases, there is very little in-band signal (green bars) reaching the sensor from the ground; even over bright targets, at least half of the in-band radiance is from the atmosphere (blue bars). For the vegetated and desert surfaces, $50 \%$ of the total radiance is from out-of-band and most is crosstalk from the SWIR1 band (red bars). Over the dark ocean there is little out-of-band radiance as the water is very dark in the other SWIR bands and over $90 \%$ of the in-band radiance is due to the atmosphere.

Figure 16. (a) Simulated Cirrus band radiances for various targets and atmospheres. The scale is expanded in order to see the small signal levels; (b) Cirrus signal plotted as a percentage of the total satellite-reaching radiance.

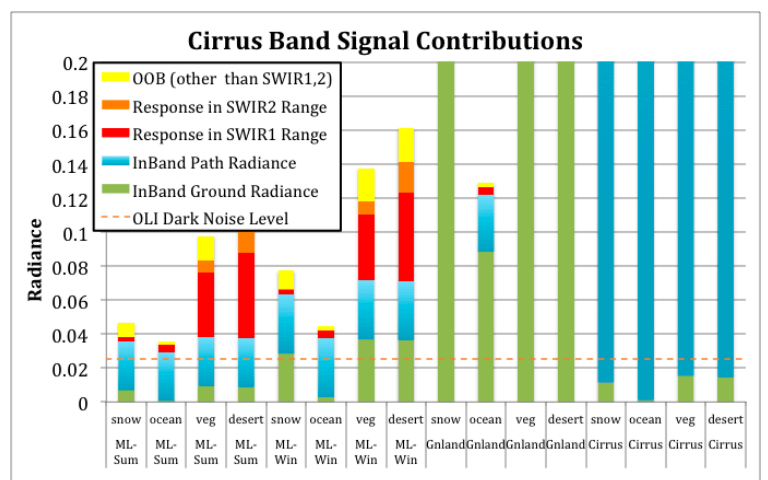

(a) Target/Atmosphere

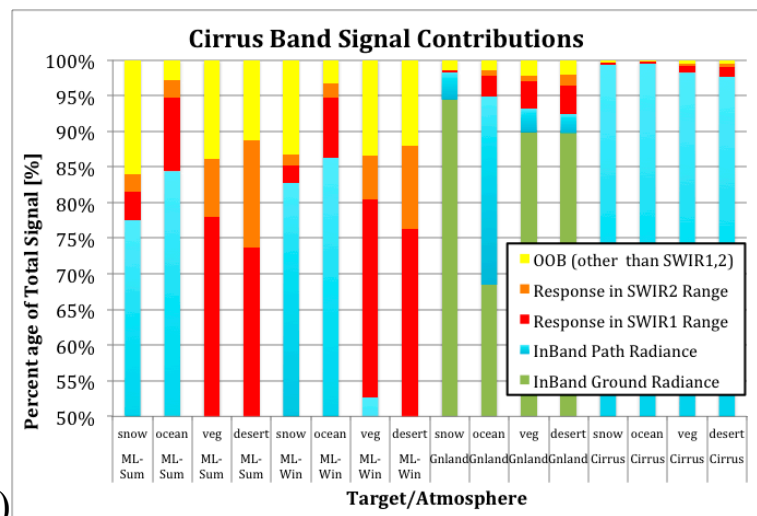


In very dry, cirrus-free atmospheres, as in the Greenland case, the in-band response over land increases due to reflected light from the surface reaching the sensor. Though there is still a cross talk component, it is less than $5 \%$ of the total response. It is important to note that under some conditions (water vapor $\leq 0.5 \mathrm{~cm}$ ) the Cirrus band will see the ground.

In the case of an atmosphere containing a cirrus cloud, the in-band radiance is primarily from the atmospheric path; the in-band radiance from the ground contributes less than $0.5 \%$ of the in-band signal. The SWIR 1 crosstalk is less than $2 \%$ of the total signal even over targets that are bright in the SWIR bands.

This crosstalk should have little effect for the user except that in operational processing, the cirrus cloud detection algorithm is a simple threshold algorithm; if the cirrus reflectance is greater than 0.02, then a pixel is flagged as cirrus in the quality band. The weak crosstalk could make an otherwise non-cirrus pixel get flagged as a cirrus pixel by pushing it over the threshold. Thus, the cirrus cloud mask will flag more cirrus clouds over targets bright in the SWIR1 band (e.g., soil and vegetation) than over targets dim in the SWIR1 (e.g., water and snow).

Similar crosstalk analysis was done for the SWIR1 and SWIR2 bands. The in-band signal for all cases is greater than $99 \%$ of the total SWIR1 signal and greater than $99.9 \%$ of the total SWIR2 signal. The contribution from crosstalk to the SWIR1 band is approximately $0.15 \%$, though other out-of-band response reached as much as $0.5 \%$.

\section{Conclusions}

The spectral response function of the OLI was well characterized during prelaunch testing and the on-orbit data are generally consistent with the prelaunch results. The in-band spectral response characteristics were measured including band edges, average and minimum response, bandpass uniformity. The uniformity results show that spectral differences between modules can result in as much as $0.35 \%$ difference in radiance between adjacent modules for a spatially uniform target when flat-fielded based on the solar diffuser data.

Once on-orbit, the crosstalk that was hinted at in the prelaunch data became apparent under specific conditions, namely in a cirrus-free atmosphere, where the surface is bright in the SWIR1 band. Though the crosstalk is visible in the Cirrus data under certain conditions (being up to $40 \%$ of the total Cirrus band signal), it is only likely to affect the cirrus cloud detection to a small extent. The crosstalk effect in the SWIR1 and SWIR2 bands is much smaller, only $0.15 \%$, and unlikely to be visible under any conditions.

The spectral response functions are published to the Landsat- 8 website. The in-band RSRs are at http://landsat.gsfc.nasa.gov/?p=5779 and the out-of-band RSRs are at http://landsat.gsfc.nasa.gov $/ ? \mathrm{p}=8829$.

\section{Acknowledgments}

The OLI was built and tested by Ball Aerospace and Technologies, Corp. All the pre-launch data discussed herein were acquired and processed by BATC personnel in order to verify compliance with OLI performance requirements. Beyond the BATC personnel included as authors, a large team was involved in designing, fabricating and testing the OLI. The authors would like to recognize the members of the Ball team involved in the data reduction including Sandra Collins, Kirk Lindahl, 
Brent Canova, Eric Donley, Brian Donley and Khurrum Ansari. Numerous other USGS, NASA and contractor personnel were involved in getting the OLI data to the calibration team for analysis. The authors would also like to thank Jim Storey for his help in understanding the spatial nature of the OLI crosstalk and reprocessing the Cirrus band data.

Science Systems and Applications, Inc. work was performed under NASA contract NNG09HP18C. Ball Aerospace and Technologies, Corp. was under NASA contract NNG07HW18C.

\section{Author Contributions}

Julia Barsi and Brian Markham were the primary contributors to this text but the tests and initial analysis were performed by Ball, specifically Geir Kvaran and Kenton Lee. Jeff Pedelty was the NASA man-on-the-ground for all the OLI tests and contributed greatly to the success of the tests.

\section{Conflicts of Interest}

The authors declare no conflict of interest.

\section{References}

1. Knight, E.J.; Kvaran, G. Landsat-8 Operational Land Imager design, characterization, and performance. Remote Sens. 2014, in press.

2. Reuter, D.C.; Richardson, C.; Pellerano, F.; Irons, J.R.; Allen, R.; Anderson, M.; Jhabvala, M.; Lunsford, A.; Montanaro, M.; Smith, R.; et al. The Thermal Infrared Sensor (TIRS) on Landsat 8: Design overview and pre-launch characterization. Remote Sens. 2014, in press.

3. Barsi, J.A.; Markham, B.L.; Pedelty, J.A. The Operational Land Imager: Spectral response and spectral uniformity. Proc. SPIE. 2011, 8153, doi:10.1117/12.895438.

4. Kvaran, G.; Markham, B.; Zalewski, E. Overview of the radiometric calibration of the Operational Land Imager (OLI). In Proceedings of the 19th Annual Conference on Characterization and Radiometric Calibration for Remote Sensing (CalCon), Logan, UT, USA, 23-26 August 2010.

5. Berk, A.; Anderson, G.; Acharya, P.; Shettle, E. MODTRAN 5.2.0.0 User's Manual; Air Force Geophysical Laboratory: Hanscom AFB, MA, USA, 2008.

(C) 2014 by the authors; licensee MDPI, Basel, Switzerland. This article is an open access article distributed under the terms and conditions of the Creative Commons Attribution license (http://creativecommons.org/licenses/by/4.0/). 